\title{
DIFFERENTIALS OF THE 2ND KIND ON A PRODUCT SURFACE
}

\author{
J.C. WILSON \\ Department of Mathematics \\ Southern Illinois University \\ Carbondale, Illinois 62901 \\ U.S.A. \\ (Received January 15, 1979)
}

ABSTRACT. This paper deals with the problems of representing an arbitrary double differential of the second kind, defined on a surface which is the topological product of two curves, in terms of the products of simple differentials of the second kind on the two curves. The curves are assumed to be non-singular and irreducible in a complex projective 2-space.

KEY WORDS AND PHRASES. Algebraic surface, product surface, algebraic curves, double differentials of the second kind, simple differentials of the second kind, Abelian differentials, genus, exterior product, polar curves, resultant.

I980 MATHEMATICS SUBJECT CLASSIFICATION CODES. 14C30, $32 J 25$.

1. INTRODUCTION.

Our purpose in this paper is to prove a theorm, stated by Lefschetz, concerning double differentials of the 2nd kind on an algebraic surface. We will employ the Picard-Lefschetz technique as described in Lefschetz [1]. 
This amounts to reducing the initial differential to a new one of the same kind, but in a more suitable form for our purposes. A precise statement will be given below. In all that follows, our ground field, denoted by $\mathrm{K}$, will be the complex numbers. Also, $\mathrm{K}(\mathrm{x}, \mathrm{y}, \ldots)$ or $\mathrm{K}[\mathrm{x}, \mathrm{y}, \ldots]$ will indicate the field of rational functions or ring of polynomials, respectively, in the complex variables $x, y, \ldots$ with coefficients in $K$.

Suppose $C_{1}$ and $C_{2}$ are two distinct, irreducible, non-singular algebraic curves, each in a complex projective 2 -space. The product surface $\Phi$, i.e., the topological product $C_{1} \times C_{2}$, is then a non-singular surface in projective 4-space. If $C_{1}$ and $C_{2}$ have affine equations $\phi(\alpha, \beta)=0$ and $\psi\left(\alpha^{*}, \beta^{*}\right)=0$, respective, then an affine model, $S$, of $\Phi$ may be thought of as the surface of pairs of points $\left(\alpha, \beta ; \alpha^{*}, \beta^{*}\right)$ in complex 4-space. We will deal primarily with an affine model in this paper. Our objective is to prove the following: "Every double differential of the 2 nd kind on $\Phi$ is reducible, modulo $\mathrm{d} \omega$, (where $\omega$ is a simple differential on $\Phi$ ), to a linear combination of products $\omega \wedge \omega^{*}$ of simple differentials of the 2nd kind, where $\omega$ belongs to $c_{1}$ and $\omega^{*}$ belongs to $C_{2}$ and neither are derived." See Lefschetz [2]. We point out that the last above statement can also be realized by using modern techniques of algebraic topology. In particular, see page 80 of Hodge and Atiyah's paper "Integrals of the 2 nd Kind on an Algebraic Variety" appearing in the Annals of Mathematics, Vo1. 62, 1955. However, we feel that the classical approach used here has merit in itself since technique ranks equally with results in algebraic geometry.

\section{SECTION 1.}

Let $R=\frac{P\left(\alpha, \beta, \alpha^{*}, \beta^{*}\right)}{Q\left(\alpha, \beta, \alpha^{*}, \beta^{*}\right)}$

be a rational function such that for fixed $\alpha$ and $\beta$ or fixed $\alpha^{*}$ and $\beta^{*}, Q$ is 
not in the ideal generated by $\phi$ or $\psi$. We assume that the double differential $\omega_{2}=\operatorname{Rd} \alpha^{*} \mathrm{~d} \alpha$ is of the 2 nd kind on $S$. Here we employ the Lefschetz definition [3], i.e., if $Q=Q_{1} Q_{2} \ldots Q_{k}$, where the $Q^{\prime}$ s are distinct, irreducible polynomials, then $\omega_{2}$ is of the 2 nd kind relative to $Q_{h}$ provided there exists a simple differential $\omega$ on $S$ such that $\omega_{2}-d \omega$ is regular at the points of intersection of $Q_{h}=0$ with $S$. Such points generally form a curve on $S$, called a polar curve of $\omega_{2}$, and we designate it by $\zeta_{h}$. The simple differential depends on the choice of $h$ and we say that $\omega_{2}$ is of the 2 nd kind on $S$ provided such an $\omega$ exists for each polar curve of $\omega_{2}$. As an analogy to Lefschetz's plane section, we employ the curve $C_{2}$ and its copies on $S$, the latter obtained by varying $\alpha$ and $\beta$ on $C_{1}$. First consider the intersection of $\zeta_{h}$ with such a "general" $\mathrm{C}_{2}$. A suitable choice of affine co-ordinates will insure that none of the polar curves pass through a point at infinity on a general $\mathrm{C}_{2}$. Also, a non-singular linear transformation will guarantee that both $\phi$ and $\psi$ contain $\beta^{d_{1}}$ and $\left(\beta^{*}\right)^{d_{2}}$, where $d_{1}$ and $d_{2}$ are the degrees of $\phi$ and $\psi$, respectively. Such co-ordinate changes do not effect the kind of differential involved. Treating $\alpha$ and $\beta$ as parameters, we designate by $P_{1}\left(\alpha, \beta, \alpha_{1}^{*}, \beta_{1} *\right)$, $\mathrm{P}_{2}\left(\alpha, \beta, \alpha_{2}{ }^{*}, \beta_{2}{ }^{*}\right), \ldots, \mathrm{P}_{\mathrm{m}}\left(\alpha, \beta, \alpha_{\mathrm{m}}{ }^{*}, \beta_{\mathrm{m}}{ }^{*}\right)$ the intersection points of $\zeta_{\mathrm{h}}$ with a general $C_{2}$. The $\alpha_{i}$ * and $\beta_{i}{ }^{*}$ 's will depend on the choice of $\alpha$ and $\beta$. Suppose $\zeta_{h}$ is a polar curve of order $n$. Then, in a neighborhood of $P_{i}$ on our general $\mathrm{C}_{2}$, the following expansion of $\mathrm{R}$ is valid:

$$
\begin{aligned}
& R=\frac{R_{-n}\left(\alpha, \beta, \alpha_{i}^{*}, \beta_{i}^{*}\right)}{\left(\alpha *-\alpha_{i}^{*}\right)^{n}}+\frac{R_{-n+1}\left(\alpha, \beta, \alpha_{i}^{*}, \beta_{i}^{*}\right)}{\left(\alpha^{*}-\alpha_{i}^{*}\right)^{n-1}} \\
& +\ldots+\frac{R_{-1}\left(\alpha, \beta, \alpha_{i}^{*}, \beta_{i}^{*}\right)}{\left(\alpha^{*}-\alpha_{i} *\right)}+\text { higher powers of }\left(\alpha *-\alpha_{i}{ }^{*}\right) .
\end{aligned}
$$

We use $\left(\alpha^{*}-\alpha_{i}^{*}\right)$ as parameter and all numerators are rational in their variables. 
LEMMA 1. There exists a simple differential $\omega_{1 \mathrm{~h}}$ on $\mathrm{S}$ such that $\omega_{2}+\mathrm{d} \omega_{1 \mathrm{~h}}$ is regular on $\zeta_{h}$.

PROOF. Define $H\left(\alpha, \beta, \alpha^{*}, \beta^{*}\right)=R \prod_{i=1}^{m}\left(\alpha^{*}-\alpha_{i}^{*}\right)^{n}$. We first will obtain an expression for $R_{-n}\left(\alpha, \beta, \alpha_{i}{ }^{*}, \beta_{i}^{*}\right)$. Let $E_{1}$ be the resultant of $Q_{h}$ and $\psi$ with respect to $\beta^{*}$ and $E_{2}$ the resultant of $E_{1}$ and $\phi$ with respect to $\beta$. $E_{2}$ may be considered as a polynomial in $\alpha^{*}$ with polynomial coefficients in $\alpha$ and as such, has the $\alpha_{i}{ }^{*}$ 's as its only zeroes. For $\alpha$ finite, this implies that the elementary symmetric functions of the $\alpha_{i}{ }^{*}$ 's are polynomials in $\alpha$. Thus $\prod_{i=1}^{m}\left(\alpha^{*}-\alpha_{i}^{*}\right)^{n}$ is a polynomial in $\alpha$ and $\alpha^{*}$. For simplicity, set $\prod_{i=1}^{m}\left(\alpha^{*}-\alpha_{i}\right)^{n}$ $=x_{h}{ }^{n}\left(\alpha, \alpha^{*}\right)$, where $x_{h}^{n}$ may be thought of as the projection of $\zeta_{h}$ on the $\left(\alpha, \alpha^{*}\right)-$ plane. It is evident that $H\left(\alpha, \beta, \alpha^{*}, \beta^{*}\right)$ is of order zero on $\zeta_{h}$. Also, $H\left(\alpha, \beta, \alpha_{i}^{*}, \beta_{i}^{*}\right)=\prod_{\substack{j=1 \\ j \neq i}}^{m}\left(\alpha_{i}^{*}-\alpha_{j}^{*}\right)^{n} \cdot R_{-n}\left(\alpha, \beta, \alpha_{i}^{*}, \beta_{i}^{*}\right)$. Hence

$$
R_{-n}\left(\alpha, \beta, \alpha_{i}^{*}, \beta_{i}^{*}\right)=\frac{H\left(\alpha, \beta, \alpha_{i}^{*}, \beta_{i}^{*}\right)}{\prod_{\substack{j=1 \\ j \neq i}}^{m}\left(\alpha_{i} *-\alpha_{j}^{*}\right)^{n}}
$$

Next we set

$$
v_{n}=\frac{1}{n-1}\left[\sum_{i=1}^{m} \frac{R_{-n}\left(\alpha, \beta, \alpha_{i}^{*}, \beta_{i}^{*}\right)}{\left(\alpha^{*}-\alpha_{i}^{*}\right)^{n-1}}\right] \text {. }
$$

Using $R_{-n}$ from (1.2), we see that $v_{n}$ not only has $\zeta_{h}$ as a polar curve but can have poles

i) at points of intersection of $\zeta_{h}$ with $\zeta_{k}, k \neq h$, for then $H$ has poles and their occurrence depends on $\alpha$ and $\beta$;

ii) at points where $\alpha$ is such that $\alpha_{i}{ }^{*}=\alpha_{j}{ }^{*}, j \neq i$;

iii) if $\alpha$ or $\beta$ is infinite. 
We can now show that $\omega_{2}+d\left[V_{n} d \alpha\right]=R d \alpha * d \alpha+d\left[V_{n} d \alpha\right]$ behaves like $R$ on $\zeta_{h}$ but with $n$ replaced by $(n-1)$. First

$$
\begin{aligned}
& \frac{R_{-n}\left(\alpha, \beta, \alpha_{i}^{*}, \beta_{i}^{*}\right)}{\left(\alpha^{*}-\alpha_{i}^{*}\right)^{n}} d \alpha^{*} d \alpha+d\left[\frac{1}{n-1} \sum_{i=1}^{m} \frac{R_{-n}\left(\alpha, \beta, \alpha_{i}^{*}, \beta_{i}^{*}\right)}{\left(\alpha^{*}-\alpha_{i}^{*}\right)^{n-1}} d \alpha\right] \\
& =\frac{R_{-n}\left(\alpha, \beta, \alpha_{i}^{*}, \beta_{i}^{*}\right)}{\left(\alpha^{*}-\alpha_{i}^{*}\right)^{n}} d \alpha^{*} d \alpha+\frac{1}{n-1}\left[\sum_{i=1}^{m} d\left(\frac{R_{-n}}{\left(\alpha^{*}-\alpha_{i}^{*}\right)^{n-1}}\right) d a\right] .
\end{aligned}
$$

A typical element of the last sum can be written as

$$
\frac{1}{\left(\alpha^{*}-\alpha_{i}^{*}\right)^{n-1}}\left[\frac{\partial R_{-n}}{\partial \alpha} d \alpha+\frac{\partial R_{-n}}{\partial \beta} d \beta\right]-(n-1) \frac{R_{-n}}{\left(\alpha^{*}-\alpha_{i}^{*}\right)^{n}} d \alpha^{*} .
$$

Since not both $\phi_{\alpha}$ and $\phi_{\beta}$ vanish, we can assume $\phi_{\beta} \neq 0$ and get $d \beta=\frac{-\phi_{\alpha}}{\phi_{\beta}} \mathrm{d} \alpha$.

Then (1.4) can be written as

$$
\begin{aligned}
& \frac{\mathrm{R}_{-\mathrm{n}}\left(\alpha, \beta, \alpha_{i}^{*}, \beta_{i}^{*}\right)}{\left(\alpha^{*}-\alpha_{i}^{*}\right)^{\mathrm{n}} \mathrm{d} \alpha * \mathrm{~d} \alpha+} \\
& +\frac{1}{\mathrm{n}-1}\left[\sum_{i=1}^{m}\left\{\frac{1}{\left(\alpha^{*}-\alpha_{i}^{*}\right)^{\mathrm{n}-1}} \cdot\left(\frac{\partial \mathrm{R}_{-\mathrm{n}}}{\partial \alpha} \mathrm{d} \alpha-\frac{\partial \mathrm{R}_{-\mathrm{n}}}{\partial \beta} \cdot \frac{\phi_{\alpha}}{\phi_{B}} \mathrm{~d} \alpha\right)-\frac{(\mathrm{n}-1) \mathrm{R}_{-\mathrm{n}}}{\left(\alpha^{*}-\alpha_{i}\right)^{\mathrm{n}}} \mathrm{d} \alpha^{*}\right\} \mathrm{d} \alpha\right] .
\end{aligned}
$$

The exterior product then gives

$$
\frac{R_{-n}}{\left(\alpha^{*}-\alpha_{i}^{*}\right)^{n}} d \alpha * d \alpha-\sum_{i=1}^{m} \frac{R_{-n}}{\left(\alpha^{*}-\alpha_{i}^{*}\right)^{n}} d \alpha^{*} d \alpha
$$

Thus, in a neighborhood of $P_{i}, R d \alpha{ }^{*} \alpha+d\left[V_{n} d \alpha\right]$ behaves like $\omega_{2}$ but with $n$ replaced by $(n-1)$. Next, we can define $a v_{n-1}$ of the same type as $v_{n}$ with $(n-1)$ in place of $n$ and $R d \alpha * d \alpha+d\left[\left(v_{n}+v_{n-1}\right) d \alpha\right]$ will begin, in some neighborhood of $P_{i}$, with the term $\frac{\hat{R}_{-n+2}}{\left(\alpha^{*}-\alpha_{i}^{*}\right)^{n-2}} \cdot$ Continuing in this manner, we will arrive at the differential $\left.R d \alpha^{*} d \alpha+d\left[v_{n}+v_{n-1}+\ldots+v_{2}\right) d \alpha\right]$, a double differential of the 2 nd kind in which the corresponding $n$ is one. 
If we still denote the coefficient of $\frac{1}{\alpha^{*}-\alpha_{1}^{*}}$ by $R_{-1}$, then since $\omega_{2}$ is of the 2nd kind, its residue relative to any polar curve must be derived, i.e., $\mathrm{R}_{-1}=\frac{\mathrm{dT}}{\mathrm{d} \alpha}$ where $\mathrm{T}\left(\alpha, \beta, \alpha_{i}{ }^{*}, \beta_{i}{ }^{*}\right)$ is a rational function on $\zeta_{\mathrm{h}}$ having poles only of type i), ii), or iii), above. See Lefschetz [4]. If we set

$$
\omega_{1 h}=\left(v_{2}+\ldots+v_{n}\right) d \alpha+\sum_{i=1}^{m} \frac{T\left(d \alpha^{*}-\frac{d \alpha_{i}^{*}}{d \alpha} d \alpha\right)}{\left(\alpha^{*}-\alpha_{i}^{*}\right)},
$$

then we can immediately show that $R d \alpha * d \alpha+d \omega_{1 h}$ is regular on $\zeta_{h}$, as follows: From the above, it is evident that $R d \alpha * d \alpha+d\left[\left(v_{2}+\ldots+v_{n}\right) d \alpha\right]$ behaves like $\omega_{2}$ relative to $\zeta_{h}$ but with the corresponding $n=1$, so we need to look only at

$$
\tau=\sum_{i=1}^{m} \frac{T\left(d \alpha^{*}-\frac{d \alpha_{i} *}{d \alpha} d \alpha\right)}{\left(\alpha^{*}-\alpha_{i}^{*}\right)} .
$$

Then,

$$
\begin{aligned}
& \mathrm{d} \tau=\left[\sum_{i=1}^{\mathrm{m}} \frac{\mathrm{dT}}{\left(\alpha^{*}-\alpha_{i}^{*}\right)}\right] \mathrm{d} \alpha^{*}-\sum_{i=1}^{\mathrm{m}} \mathrm{d}\left[\frac{\mathrm{d} \alpha_{i}{ }^{*}}{\mathrm{~d} \alpha} \cdot \frac{\mathrm{T}}{\alpha^{*}-\alpha_{1}^{*}}\right] \mathrm{d} \alpha= \\
& {\left[\sum_{i=1}^{m} \frac{\left(\alpha^{*}-\alpha_{i}^{*}\right) \mathrm{dT}-\operatorname{Td}\left(\alpha^{*}-\alpha_{i}^{*}\right)}{\left(\alpha^{*}-\alpha_{i}^{*}\right)^{2}}\right] \mathrm{d} \alpha^{*}-} \\
& \left\{\sum_{i=1}^{m}\left[\frac{d \alpha_{i}^{*}}{d \alpha} \cdot d\left(\frac{T}{\left(\alpha^{*}-\alpha_{i}^{*}\right)}\right)+\frac{T}{\left(\alpha^{*}-\alpha_{i}^{*}\right)} \cdot d\left(\frac{d \alpha_{i}^{*}}{d \alpha}\right)\right]\right\} d \alpha= \\
& \sum_{i=1}^{m} \frac{\frac{d T}{d \alpha}}{\left(\alpha^{*}-\alpha_{i}^{*}\right)} d \alpha d \alpha^{*}-\sum_{i=1}^{m} \frac{T\left(d \alpha^{*}-d \alpha_{i}^{*}\right) d \alpha^{*}}{\left(\alpha^{*}-\alpha_{i}^{*}\right)^{2}}- \\
& \sum_{i=1}^{m} \frac{\mathrm{d} \alpha_{i} *}{\mathrm{~d} \alpha}\left[\frac{\left(\alpha^{*}-\alpha_{i}^{*}\right) \mathrm{dT}-\operatorname{Td}\left(\alpha^{*}-\alpha_{i}^{*}\right)}{\left(\alpha^{*}-\alpha_{i}^{*}\right)^{2}}\right]= \\
& \sum_{i=1}^{m} \frac{\frac{d T}{d \alpha}}{\left(\alpha^{*}-\alpha_{i}^{*}\right)} d \alpha d \alpha *+\sum_{i=1}^{m} \frac{T\left(\frac{d \alpha_{i}^{*}}{d \alpha}\right)}{\left(\alpha^{*}-\alpha_{i}^{*}\right)^{2}} d \alpha d \alpha *-
\end{aligned}
$$




$$
\begin{aligned}
& \sum_{i=1}^{m} \frac{\left(\frac{d \alpha_{i}{ }^{*}}{d \alpha}\right) d T}{\left(\alpha *-\alpha_{i}^{*}\right)} d \alpha d \alpha *+\sum_{i=1}^{m} \frac{\left(\frac{d \alpha_{i}{ }^{*}}{d \alpha}\right) T}{\left(\alpha^{*}-\alpha_{i}\right)^{2}} d \alpha \star d \alpha= \\
& \sum_{i=1}^{m} \frac{\frac{d T}{d \alpha}}{\alpha *-\alpha_{i}{ }^{*}} d \alpha d \alpha *,
\end{aligned}
$$

since the third term in the last sum above is zero and the second and fourth terms are negatives of one another. However,

$$
\sum_{i=1}^{m} \frac{\frac{d T}{d \alpha}}{\alpha^{*}-\alpha_{i}^{*}} d \alpha d \alpha *=-\sum_{i=1}^{m} \frac{R_{-1}}{\alpha^{*}-\alpha_{i}^{*}} d \alpha \star d \alpha .
$$

This concludes the proof of Lemma 1 .

For brevity, we will write $\omega_{1 h}$ in $(1.5)$ as

$$
\omega_{1 h}=V(\alpha, \beta, \alpha *, \beta *) d \alpha-U(\alpha, \beta, \alpha *, \beta *) d \alpha *
$$

where $\mathrm{V}$ and $\mathrm{U}$ are rational on $\mathrm{S}$. From Lemma $1, \mathrm{~V}$ and $\mathrm{U}$ are infinite on the intersection of $X_{h}=0$ with $\mathrm{S}$, so that in addition to $\zeta_{h}$, they are infinite on any other curve which projects onto $\chi_{h}=0$. We will designate this residual intersection by $\mathrm{D}_{h}$.

LEMMA 2. $\omega_{1 h}$ may be replaced with a similar differential but with $D_{h}$ eliminated as a polar curve (or curves).

PROOF. For almost any non-singular affine transformation $(\alpha, \beta, \alpha *, \beta *)$ to $(\tilde{\alpha}, \tilde{\beta}, \tilde{\alpha} *, \tilde{\beta} *)$, the projections of the transforms of $\zeta_{h}$ and $D_{h}$ on the

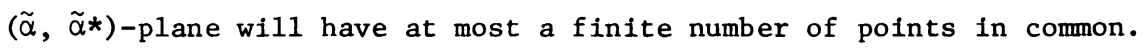
Under such a transformation $R d \alpha \star d \alpha+d(V d \alpha-U d \alpha *)$ becomes

$$
\tilde{\mathrm{R}} \mathrm{d} \tilde{\alpha} * \mathrm{~d} \tilde{\alpha}+\mathrm{d}\left(\tilde{\mathrm{V}} \mathrm{d} \tilde{\alpha}-\tilde{\mathrm{U}} \mathrm{d} \tilde{\alpha}{ }^{*}\right) \text {, }
$$

where we set

$$
\tilde{V}=\frac{\tilde{A}(\tilde{\alpha}, \tilde{\beta}, \tilde{\alpha} *, \tilde{\beta} *)}{\tilde{G}(\tilde{\alpha}, \tilde{\beta}, \tilde{\alpha} *, \tilde{\beta} *)} \text { and }
$$




$$
\tilde{U}=\frac{\tilde{B}\left(\tilde{\alpha}, \tilde{\beta}, \tilde{\alpha} *, \tilde{\beta}^{*}\right)}{\tilde{G}\left(\tilde{\alpha}, \tilde{\beta}, \tilde{\alpha}^{\star}, \tilde{\beta}^{*}\right)},
$$

$\tilde{A}, \tilde{B}$, and $\tilde{G}$ polynomials. Here we have made use of the transformations $\phi(\alpha, \beta)$ to $\tilde{\phi}\left(\tilde{\alpha}, \tilde{\beta}, \tilde{\alpha}^{\star}, \tilde{\beta}^{*}\right)$ and $\psi\left(\alpha^{*}, \beta^{*}\right)$ to $\tilde{\psi}\left(\tilde{\alpha}, \tilde{\beta}, \tilde{\alpha} *, \tilde{\beta}^{*}\right)$ in eliminating $\mathrm{d} \tilde{\beta}$ and $d \tilde{\beta} *$. We can also assume that neither $\frac{\partial \tilde{\phi}}{\partial \tilde{\beta}}$ nor $\frac{\partial \tilde{\psi}}{\partial \tilde{\beta}^{*}}$ are zero, due to the absence of singularities. Let the resultant of $\tilde{G}$ and $\tilde{\psi}$ with respect to $\tilde{\beta}^{*}$ be denoted by $\tilde{E}_{1}$, i.e., I $\tilde{G}+J \tilde{\psi}=\tilde{E}_{1}$. Further, the resultant of $\tilde{E}_{1}$ and $\tilde{\phi}$ with respect to $\tilde{\beta}$ gives a second resultant, say $G_{1}$, i.e., $L \tilde{E}_{1}+M \tilde{\phi}=G_{1}$. Thus, $L I \tilde{G}+L J \tilde{\psi}=G_{1}-M \tilde{\phi}$ and in the transformed surface LIE $=G_{1}$ since $L J \tilde{\psi}$ and $M \tilde{\phi}$ are zero. It follows that $\omega_{1 \mathrm{~h}}$ can be written as

$$
\omega_{1 h}=\frac{\tilde{\tilde{A}}(\tilde{\alpha}, \tilde{\beta}, \tilde{\alpha} *, \tilde{\beta} *)}{G_{1}\left(\tilde{\alpha}, \tilde{\alpha}^{*}\right)} \mathrm{d} \tilde{\alpha}-\frac{\tilde{\tilde{B}}\left(\tilde{\alpha}, \tilde{\beta}, \tilde{\alpha^{*}}, \tilde{\beta} *\right)}{G_{1}\left(\tilde{\alpha}, \tilde{\alpha}^{*}\right)} \mathrm{d}^{*} \text {, }
$$

where

$$
\tilde{\tilde{A}}=\operatorname{LIA}, \quad \tilde{\tilde{B}}=\cdot \operatorname{LI} \tilde{B}
$$

and both are polynomials. If we call the projection of the transform of $\zeta_{h}$, in the $\left(\tilde{\alpha}, \tilde{\alpha}^{*}\right)$-plane, by $\tilde{\chi}_{h}$, then $G_{1}=\tilde{\chi}_{h}{ }^{n}\left(\tilde{\alpha}, \tilde{\alpha}^{*}\right) \tilde{\tilde{\chi}}_{h}\left(\tilde{\alpha}, \tilde{\alpha}^{*}\right)$, where $\tilde{\chi}_{h}{ }^{n}$ and $\tilde{\tilde{\chi}}_{h}$ are relatively prime polynomials. Considering them as polynomials in $\tilde{\alpha}^{*}$ with coefficients in $\tilde{\alpha}$, there exist polynomials $s\left(\tilde{\alpha}, \tilde{\alpha}^{*}\right)$ and $t\left(\tilde{\alpha}, \tilde{\alpha}^{*}\right)$ such that

$$
s \tilde{\chi}_{h}^{n}+t \tilde{\tilde{\chi}}_{h}=p(\tilde{\alpha})
$$

Using this last equality, we can write

$$
\frac{1}{G_{1}}=\frac{1}{\tilde{X}_{h}^{n} \tilde{\chi}_{h}}=\frac{1}{p(\tilde{\alpha})}\left[\frac{s}{\tilde{\tilde{X}}_{h}}+\frac{t}{\tilde{X}_{h}{ }^{n}}\right]=\frac{t}{p(\tilde{\alpha}) \tilde{X}_{h}{ }^{n}}+\frac{s}{P(\tilde{\alpha}) \tilde{\tilde{X}}_{h}} .
$$

Therefore 


$$
\begin{aligned}
& \omega_{1 h}=[\tilde{\tilde{A}} d \tilde{\alpha}-\tilde{\tilde{B}} d \tilde{\alpha} *] \cdot\left[\frac{t}{p(\tilde{\alpha}) \tilde{\chi}_{h}^{n}}+\frac{s}{p(\tilde{\alpha}) \tilde{\tilde{\chi}}_{h}}\right]= \\
& \tilde{\tilde{A}}\left[\frac{t}{p(\tilde{\alpha}) \tilde{X}_{h}^{n}}+\frac{s}{p(\tilde{\alpha}) \tilde{X}_{h}}\right] d \tilde{\alpha}-\tilde{\tilde{B}}\left[\frac{t}{p \tilde{X}_{h}^{n}}+\frac{s}{p \tilde{\tilde{X}}_{h}}\right] d \tilde{\alpha}^{*}= \\
& \frac{\tilde{\tilde{A}} t d \tilde{\alpha}-\tilde{\tilde{B}}_{t} \mathrm{~d} \tilde{\alpha} *}{\mathrm{p}(\tilde{\alpha}) \tilde{\chi}_{\mathrm{h}}{ }^{\mathrm{n}}}+\frac{\tilde{\tilde{A}} s \mathrm{~d} \tilde{\alpha}-\tilde{\tilde{B}} s \mathrm{~d} \tilde{\alpha} *}{\mathrm{p}(\tilde{\alpha}) \tilde{\tilde{\chi}}_{\mathrm{h}}}
\end{aligned}
$$

It is evident that $\tilde{R} d \tilde{\alpha} \star d \tilde{\alpha}+d \omega_{1 h}$ is regular on the transform of $\underset{\tilde{\tilde{\alpha}}}{\zeta_{h}}$ and this is true even if we suppress the last term above, i.e., $\frac{\tilde{\tilde{A}} s \mathrm{~d} \tilde{\alpha}-\tilde{\tilde{B}} \mathrm{sd} \tilde{\alpha}}{\mathrm{p}} \tilde{\tilde{X}}_{\mathrm{h}}$ If we replace $\omega_{1 h}$ with $\tilde{\omega}_{1 h}=\frac{\tilde{\tilde{A}} t d \tilde{\alpha}-\tilde{\tilde{B}}_{t d \tilde{\alpha}^{\star}}}{p(\tilde{\alpha}) \tilde{\chi}_{h}^{n}}=\frac{A_{h} d \tilde{\alpha}-B_{h} d \tilde{\alpha}^{\star}}{p(\tilde{\alpha}) \tilde{X}_{h}^{n}}$, we have $\tilde{R}^{n} \tilde{\alpha}^{\star} d \tilde{\alpha}+d \tilde{\omega}_{1 h}$, a differential of the 2 nd kind on the transformed surface, regular on $\zeta_{h}$ transform and the transform of $D_{h}$ is no longer a polar curve of $\tilde{\omega}_{1 h}$.

\section{SECTION 2 .}

The above reduction and replacement can be carried out for all polar curves $\zeta_{\mathrm{h}}$ of $\omega_{2}$. Returning to the original notation and co-ordinates, we would then have a set of $\omega_{1 h}$ such that $\omega_{2}+d\left(\sum_{h} \omega_{1 h}\right)$ would be regular on the $\zeta_{h}$ but would have poles for certain values of $\alpha$ and $\beta$. Let us write $\omega_{2}+d\left(\sum_{h} \omega_{1 h}\right)$ in the form

$$
\mathrm{w}_{2}=\frac{A\left(\alpha, \beta, \alpha^{*}, \beta^{*}\right) \mathrm{d} \alpha^{*} \mathrm{~d} \alpha}{B\left(\alpha, \beta, \alpha^{*}, \beta^{*}\right) G(\alpha)}
$$

where $A, B$, and $G$ are polynomials and $A / B$ is regular except possibly for points of infinity.

LEMMA 3. $\mathrm{W}_{2}=\frac{A \mathrm{~d} \alpha * \mathrm{~d} \alpha}{B G}$ can be reduced to the form $\frac{\pi(\alpha, \beta, \alpha *, \beta *)}{G(\alpha)} \mathrm{d} \alpha * \mathrm{~d} \alpha$ where $\pi$ is a polynomial.

PROOF. We begin by replacing the affine co-ordinates $\alpha$ * and $\beta$ * with projective co-ordinates $\alpha_{0}{ }^{*} \alpha_{1} *$, and $\alpha_{2}$ in both $\psi\left(\alpha *, \beta^{*}\right)$ and $B\left(\alpha, \beta, \alpha^{*}, \beta^{*}\right)$ where $B$ is to be regarded as a polynomial in $\alpha_{0} *, \alpha_{1} *, \alpha_{2} *$ with coefficients 
in the field $K(\alpha, \beta)$. By Kapferer's Theorem [5], $A$ is representable in the form $\mathrm{Y} \psi+Z B, Y$ and $Z$ polynomials in $\alpha_{0}{ }^{*}, \alpha_{1}{ }^{*}$, and $\alpha_{2}{ }^{*}$. This is true since $A$ must vanish at the simultaneous zeros of $\psi$ and $B$ with a multiplicity at least as great as $B$ and any such zero of $\psi$ and $B$ is a finite simple point of $\psi$. Thus, $A=\mathrm{Y} \psi+\mathrm{Z} B$ and on the surface, $A=\mathrm{Z} B$, so that $A / B=\mathrm{Z}$, a polynomial in $\alpha_{0} *, \alpha_{1}{ }^{*}, \alpha_{2}$ * with coefficients in $\mathrm{k}(\alpha, \beta)$. Returning to affine co-ordinates, we see that the ratio $A / B$ is a polynomial in $\alpha^{*}, \beta^{*}$ with rational coefficients in $\alpha$ and $\beta$ so that we can write

$$
\frac{A}{B}=\frac{F\left(\alpha, \beta, \alpha^{*}, \beta^{*}\right)}{D(\alpha, \beta)}
$$

where $F$ and $D$ are polynomials. Let $E$ be the resultant with respect to $\beta$ of $D$ and $\phi(\alpha, \beta)$, i.e., $E=\gamma \phi+\delta D$. Therefore,

$$
\frac{F}{D}=\frac{\delta F}{\delta D}=\frac{\delta F}{E-\gamma \phi}
$$

and on the surface

$$
\frac{F}{D}=\frac{\delta F}{E}=\frac{\tilde{F}}{E}
$$

If $\alpha=\alpha_{0}$ is a zero of order $\mathrm{r}$ of $E$, then $\tilde{F}$ must be divisible by $\left(\alpha-\alpha_{0}\right)^{\mathrm{r}}$ since $A / B$ has no poles for $\alpha$ finite. Thus, $\tilde{F} / E$ is a polynomial $\pi\left(\alpha, \beta, \alpha^{*}, \beta^{*}\right)$ on S. Finally, we can write

$$
\mathrm{W}_{2}=\frac{\pi \mathrm{d} \alpha * \mathrm{~d} \alpha}{G(\alpha)}
$$

and $\mathrm{W}_{2}$ has poles only at the zeroes of $G$ and possibly $\alpha=\infty$. We have arrived at our final reduced form for $\omega_{2}$, i.e., $(\pi / G) \mathrm{d} \alpha \star d \alpha$ (modulo $d \omega_{1}$ ), whose only polar curves on $S$ are $C_{2}$ or its copies. Any double differential of the 2nd kind may, by subtraction of a suitable $d \omega_{1}$, be reduced to one of the form (2.1).

THEOREM. Any double differential of the 2nd kind on $\Phi$ can be reduced, 
modulo $d \omega_{1}$, to the form $\sum c_{i j} \omega_{i} \wedge \omega_{j}$ * where $\omega_{i}$ and $\omega_{j}{ }^{*}$ are simple differentials of the 2nd kind on $c_{1}$ and $c_{2}$ and no $\omega_{i}$ or $\omega_{j}^{*}$ is derived.

PROOF. We begin by writing $\omega_{2}$ in the form (2.1), i.e.,

$$
\omega_{2}=\frac{\pi}{G} \mathrm{~d} \alpha * \mathrm{~d} \alpha
$$

If $\pi$ is of degree $d$ in $\beta^{*}$, we can write

$$
\begin{aligned}
\pi= & c_{d}\left[\alpha, \beta, \alpha^{*}\right]\left(\beta^{*}\right)^{d}+c_{d-1}\left[\alpha, \beta, \alpha^{*}\right]\left(\beta^{*}\right)^{d-1} \\
& +\ldots+c_{1}\left[\alpha, \beta, \alpha^{*}\right] \beta^{*}+c_{0}\left[\alpha, \beta, \alpha^{*}\right],
\end{aligned}
$$

where the c's are polynomials. Also, each of the c's can be written as a polynomial in $\alpha^{*}$. Let the degree of $c_{i}$ in $\alpha^{*}$ be $d_{i}$. Then, each $c_{i}$ can be written

$$
c_{i}=b_{i, d_{i}}\left(\alpha^{*}\right)^{d_{i}}+b_{i, d_{i}-1}\left(\alpha^{*}\right)^{d_{i}-1}+\ldots+b_{i, 1} \alpha^{*}+b_{i, 0},
$$

where the $b^{\prime}$ 's are polynomials in $\alpha$ and $\beta$. It is then clear that $\pi$ can be written as a finite sum of terms of the form

$$
\frac{P(\alpha, \beta)\left(\alpha^{*}\right)^{\mathrm{p}}\left(\beta^{*}\right)^{\mathrm{q}}}{G(\alpha)}
$$

where $P$ is a polynomial and $p$ and $q$ are positive integers. Consider

$$
\frac{P(\alpha, \beta)\left(\alpha^{*}\right)^{\mathrm{P}}\left(\beta^{*}\right)^{\mathrm{q}}}{G(\alpha)} \mathrm{d} \alpha * \mathrm{~d} \alpha
$$

This is a double differential of the 2 nd kind on $S$ and can be written as

$$
\left[\left(\alpha^{*}\right)^{\mathrm{p}}\left(\beta^{*}\right)^{\mathrm{q}} \mathrm{d} \alpha^{*}\right]\left[\frac{P(\alpha, \beta) \mathrm{d} \alpha}{G(\alpha)}\right],
$$

a product of two Abelian differentials, the first on $C_{2}$ and the second on $C_{1}$. Each of them must be of the 2nd kind on their respective curves since, 
if not, the double differential would have non-zero residues on $S$. Let the genus of $C_{1}$ be $g_{1}$ and that of $C_{2}$ be $g_{2}$. Also, let $d_{\mu_{1}}, d_{\mu_{2}}, \ldots, d_{\mu_{2 g_{1}}}$ and ${ }^{d} v_{1}, d_{v_{2}}, \ldots, d_{v_{2 g}}$ be bases for differentials of the 2 nd kind on $c_{1}$ and $c_{2}$, respectively. Then

$$
\left(\alpha^{*}\right)^{p}\left(\beta^{*}\right)^{q} d \alpha^{*}=\sum_{1}^{2 g_{2}} \tilde{c}_{k} d \nu_{k}
$$

and

$$
\frac{P \mathrm{~d} \alpha}{G}=\sum_{1}^{2 g_{1}} c_{i} \mathrm{~d} \mu_{i},
$$

where the c's are constant. Thus,

$$
\begin{aligned}
& \frac{P(\alpha, \beta)}{G(\alpha)}\left(\alpha^{*}\right)^{p}\left(\beta^{*}\right)^{q} \mathrm{~d} \alpha^{*} \mathrm{~d} \alpha= \\
& \sum_{i=1}^{2 g_{1}}\left[\sum_{k=1}^{2 g_{2}} c_{i k} d \nu_{k} d \mu_{i}\right]
\end{aligned}
$$

where $c_{i k}=c_{i} \tilde{c}_{k}$. Since each term in $\pi / G d \alpha \star d \alpha$ can be so written, the theorem follows.

\section{REFERENCES}

1. Lefschetz, Solomon. Algebraic Geometry, Princeton University Press, 1953, p. $196-210$.

2. Ibid, p. 221.

3. Ibid, p. 202 .

4. Ibid, p. 206.

5. Van der Waerden, B. L. Einfuehrung in die Algebraische Geometrie, Springer, Berlin, 1939, p. 209. 


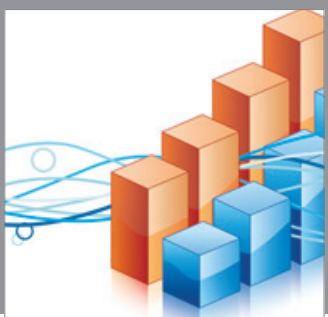

Advances in

Operations Research

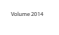

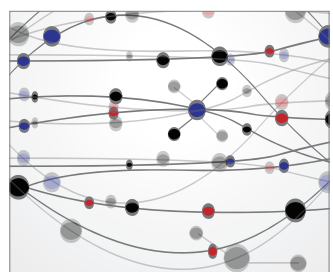

\section{The Scientific} World Journal
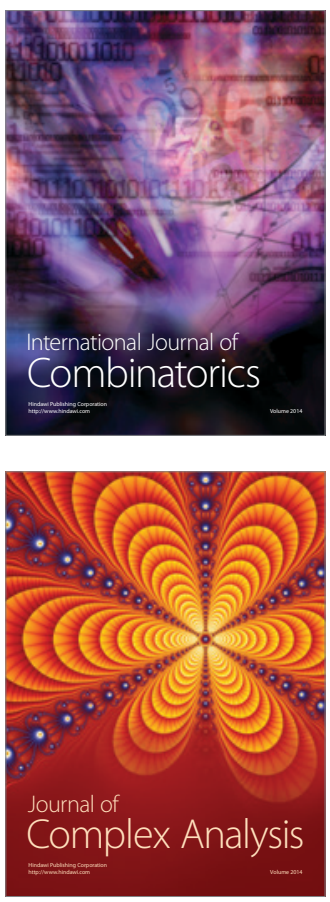

International Journal of

Mathematics and

Mathematical

Sciences
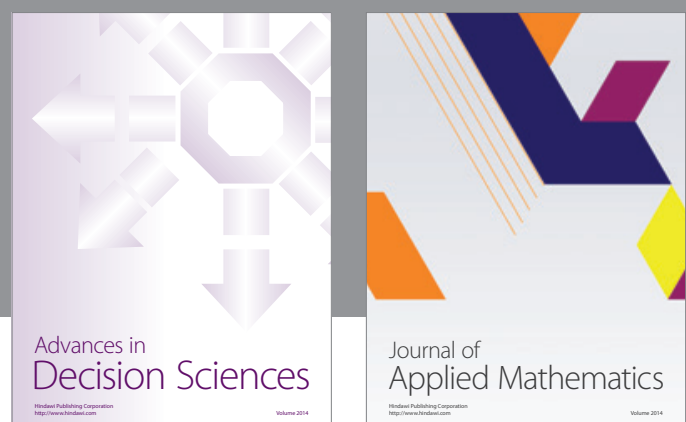

Journal of

Applied Mathematics
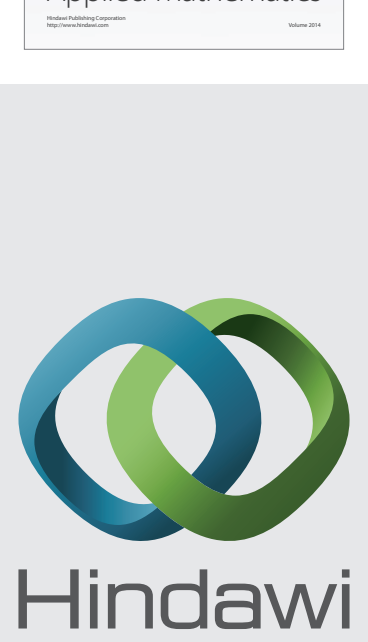

Submit your manuscripts at http://www.hindawi.com
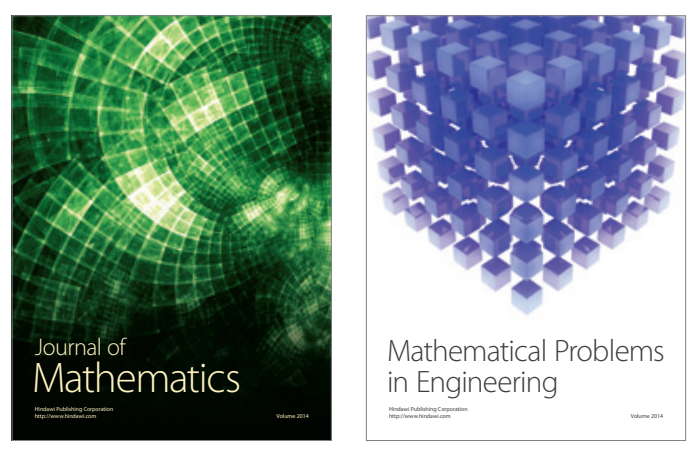

Mathematical Problems in Engineering
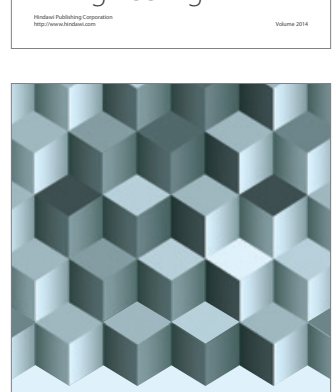

Journal of

Function Spaces
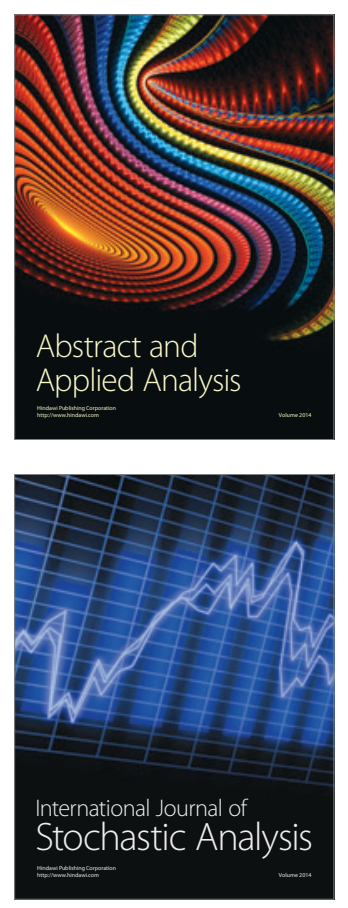

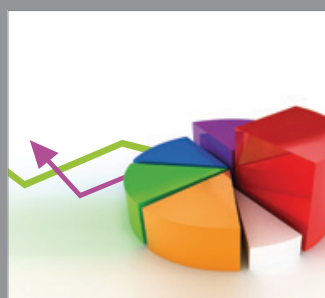

ournal of

Probability and Statistics

Promensencen
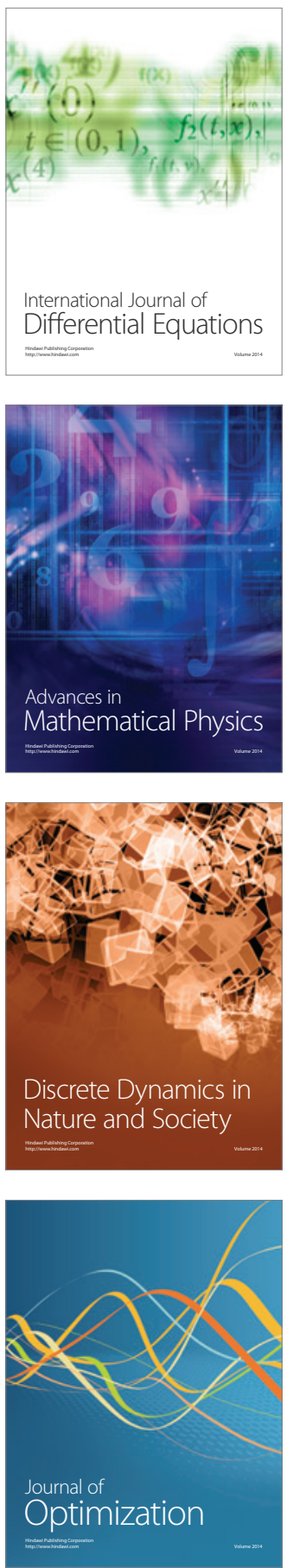